\title{
Carence en cobalt dans un élevage de bovidés au Katanga
}

\author{
par Hans E. SUTER \\ Au professeur R. GEIGY \\ à l'occasion de son 60 e anniversaire.
}

\section{INTRODUCTION}

La nutrition des ruminants est hautement influencée par le cobalt, oligo-élément utilisé par la flore du rumen pour synthétiser la cyanocobalamine ou vitamine $B_{12}$. Cette dernière, stockée dans le foie des ruminants, contient du cobalt trivalent. Le dépôt de vitamine $B_{12}$ dans le foie varie proportionnellement à l'apport alimentaire de cobalt.

Les observations de carence en cobalt dans l'alimentation des animaux se sont multipliées durant ces quinzes dernières années et proviennent de tous les continents (cf. bibliographie). Cette carence a été observée en NouvelleZélande, en Australie, aux Etats-Unis, au Canada, au Brésil, au Kenya, aux Hébrides ; en Europe, en Irlande, en Ecosse, en Angleterre. La Norvège, le Danemark, la Suède et l'Allemagne ne sont pas épargnés. L'appellation de la carence en cobalt est aussi variée que les pays où elle fut signalée.

« Bush sickness » en Nouvelle-Zélande,

« nakuruitis » au Kenya,

« enzootic marasmus » ou « cost disease » en Australie,

« Danmark wasting disease » au Danemark représentent des entités à étiologie commune.

Les recherches quantitatives sur la teneur en cobalt des herbes de pâturages normaux et de pâturages carencés n'ont pas permis de con-

Rev. Elev. Med. vét. Pays trop., 1962, 15, nº 1.

Reçu pour publication : décembre 1961. clusions nettement définies pour classer les différents pâturages. BEESON (d'après GEE) (2) signale que des taux de 0,03 à $0,14 \mathrm{mg}$ par $\mathrm{kg}$ de matières sèches de foin récolté ont été trouvés dans des pâturages déficients, alors que des taux de 0,07 à $0,26 \mathrm{mg}$ se rencontrent dans des pâturages normaux. Les valeurs trouvées dans les pâturages suisses varient de 0,07 à $0,42 \mathrm{mg}$ (A. HASLER et R. ZUBER) (4). On observe d'autre part des variations dans les mêmes pâturages au courant de la même année et également d'une année à l'autre.

Suivant HASLER et ZUBER (4) les besoins en cobalt ne seraient pas constants ef varieraient avec la teneur en lignine et en cellulose du fourrage.

On peut toutefois situer la limite inférieure du taux de cobalt dans un fourrage normal aux environs de $0,07 \mathrm{mg}$ par $\mathrm{kg}$ de matière sèche (BEESON, d'après GEE) (2).

La symptomatologie de la carence en cobalt chez les ruminants se résume à une perte de l'appétit, pica, amaigrissement, : poil rêche, anémie, diarrhée.

La mortalité des veaux, nés de mères carencées, est élevée au cours des premières semaines de vie.L'évolution de la maladie simule une sousalimentation prolongée et la pathogénie s'expliquerait par la diminution de l'action digestive de la flore du rumen.

Les déficiences subcliniques ou intermittentes revêtent une grande importance dans de nombreuses régions; elles peuvent être révélées par l'action favorable de l'adjonction de cobalt à l'alimentation (épreuve thérapeutique). 


\section{OBSERVATIONS PERSONNELLES}

Les observations rapportées ont élé faites au cours des années 1946 à 1956, dans un grand élevage de bovidés dcstinćs uniquement à la boucherie. La région se situe sous le 7 e degré de latitude sud, donc en zone dite équatoriale. L'altitude de 1.100 mètres modifie les conditions de température et crée des conditions climatiques particulières: une saison sèche et relativement froide de 4 à 5 mois et une saison des pluies d'environ 7 mois, avec des précipitations variant de 1.200 à $1.500 \mathrm{~mm}$ par an.

Le bétail est élevé uniquement dans des conditions de ranching extensif, sans supplément de nourriture. Il reçoit uniquement et à volonté du sel une fois par semaine, après le bain antiparasitaire hebdomadaire.

On compte 5 à 6 hectares de pâturage par tête. La vigoureuse poussée des herbes en saison des pluies oblige de tenir le bétail sur des espaces relativement restreints ; cela permet de maintenir les herbes courtes et d'éviter leur trop forte poussée, suivie inévitablement par une rapide transformation cellulosique. Pour procurer au bétail des pâturages convenables, il est nécessaire de brûler les vieilles herbes sans valeur nutritive, selon un système rigoureux de planning préétabli ; dans la deuxième moitié de la saison des pluies ef au début de la saison sèche, de grandes superficies sont ainsi préparées pour servir de pâturage pendant la deuxième partie de la saison des pluies et les 4 à 5 mois de saison sèche; les jeunes pousses remplacent les vieux. herbages trop riches en cellulose, en se procurant l'humidité à partir des réserves accumulées dans le sol.

Le pays se présente sous forme de grandes plaines herbeuses, entrecoupées de galeries forestières de faible largeur ou de vallonnements non boisés, larges et marécageux.

Le terrain est sablonneux, argilo-sableux ou argileux. Souvent les zones argileuses sont colorées en rouge par la présence de limonite, alors que les autres formations varient du rouge clair au blane grisâtre.

Le comportement du bétail varie en fonction du type de terrain de certaines régions; cette différence de comportement fut particulièrement nette dans les zones étudiées ici. Les bêtes pâturant sur les terrains rouges, riches en limo- nite, supportent très bien les deux saisons, alors que celles pâturant sur les terrains clairs ne se maintiennent en bonne santé que jusque vers la moitié de la saison des pluies; après cette période, l'état général décline, pour arriver d son point le plus bas à la fin de la saison des pluies. C'est à ce moment que le taux de mortalité s'élève ; les survivants ont besoin de toute la première moitié de la saison sèche pour se remettre. Le même cycle se répète d'une année à l'autre, indépendamment de l'âge des animaux. Quand les malades furent placés sur des terrains riches en limonite, ils guérirent sans aucune médication.

La symplomatologie observée fut la suivante :

१. Perte d'appétit. Entre 9-10 h. du matin, les bêtes cessent de brouter. Elles recherchent l'ombre ou se rassemblent en groupe, têtes baissées, oreilles pendantes. Tout effort pour séparer les bêtes rassemblées reste sans succès. Les animaux ne recommencent à paître que vers $16 \mathrm{~h}$. Ce comportement ne varie. même pas, si les pâtures sont couvertes de jeunes pousses et présentent un excellent aspect.

2. Pica. Le bétail lèche la terre rouge des termitières et ronge l'écorce des arbustes, mâche des branches, des pierres, des os. II délaisse le sel ordinaire donné chaque semaine; cette abstinence envers le sel est proportionnelle à l'augmentation du pica.

3. Amaigrissement. Le poil devient rêche, l'apparence de tristesse augmente, le ventre se creuse les animaux restent immobiles, le dos légèrement voûté, tandis que l'amaigrissement s'accentue progressivement.

4. Anémie. Sans que les numérations globulaires aient été pratiquées, les nombreux frottis ont cependant mis en évidence un sang clair, avec un nombre d'érythrocytes nettement diminué et une anisocytose marquée.

5. Diarrhées. Souvent, mais non toujours, la chute de poids s'accélère avec l'apparition de diarrhée ; les animaux se couchent et meurent cachectiques.

6. Complications. Cette image clinique se complique fréquemment de toutes sortes d'affections intercurrentes, qui parviennent facilement à se greffer sur des animaux dont l'état général est profondément atteint. Nous avons observé 


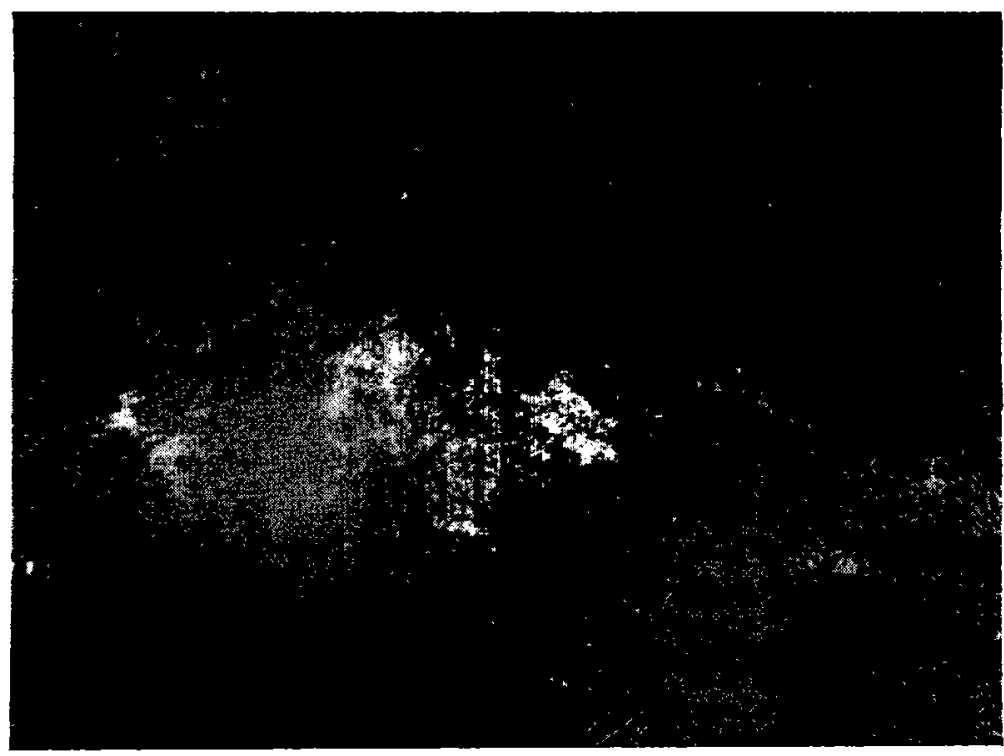

Photo 1. - Termitière léchée par le bétail malade.

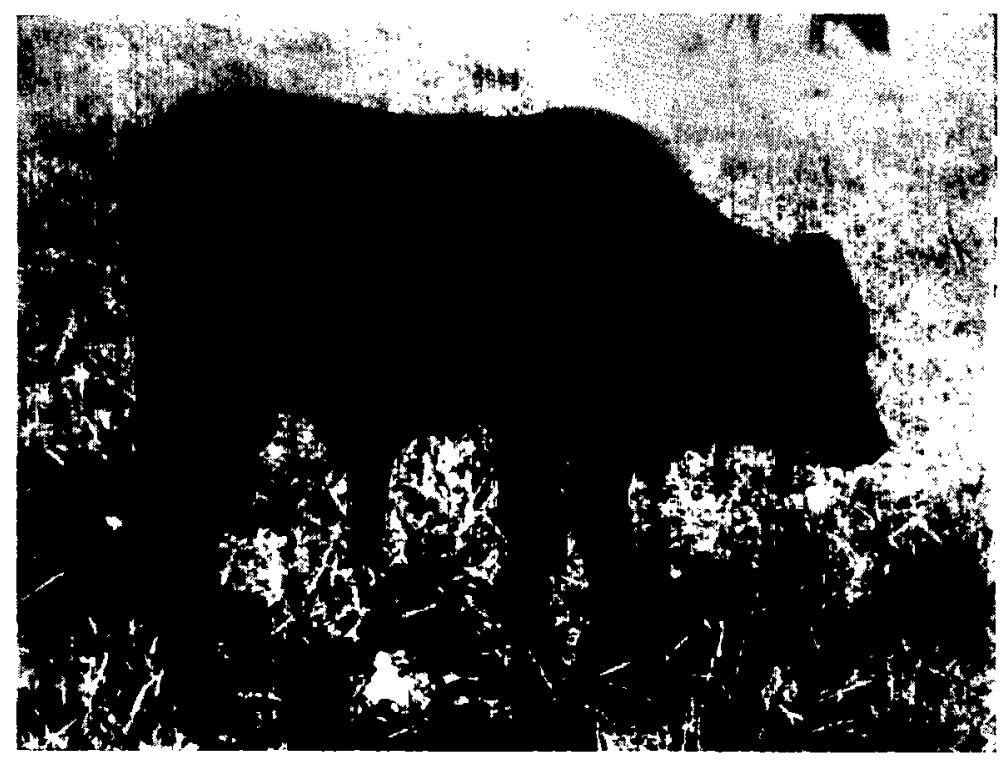

Photo 2. - Bouvillon d'un an et demi présentant les premiers symptômes de la maladie. 
en particulier des infestations parasitaires (trichostrongylidés, bunostomes), des dermatites graves, du coryża gangréneuux (avec nécrose de la muqueuse du mufle se décollant par plaques), des ulcérations de la muqueuse buccale, de la langue, de la caillette et du duodénum, du larmoiement avec conjonctivite et même des kératites graves.

Les constatations nécropsiques n'apportent aucun élément nouveau :

Fonte du pannicule adipeux.

Pâleur des tissus.

Sang clair, aqueux, non-coagulé.

Augmentation du liquide des cavités séreuses.

Hypertrophie muqueuse de l'intestin grêle, avec œè̀me et enduit épais, blanchâtre, mucopurulent.

Foie de couleur gris pâle ; parenchyme friable. d'aspect granulaire à la coupe.

Myocarde pâle avec souvent pétéchies sousépicardiques et sous-endocardiques.

Hypertrophie ganglionnaire généralisée avec stase séreuse; rate normale.

A cette image s'ajoutent les lésions causées par les maladies concomitantes créant un polymorphisme lésionnel, fonction surtout de la variété des affections intercurrentes.

\section{HYPOTHESSE DE TRAVAIL}

La symptomatologie décrite atteignait, certaines années, des centaines de bêtes ; longtemps elle fut considérée comme l'expression d'une maladie d'étiologie inconnue. Les parasitoses intestinales furent envisagées au début; pour diminuer les possibilités d'infestation, des mesures strictes d'hygiène des pâturages et des abreuvoirs furent appliquées. De plus, différents vermifuges furent administrés. Une solution de sulfate de cuivre et d'arséniate de soude avait particulièrement retenu l'attention. La rotation des pâturages fut accélérée et les troupeaux ne pâturaient que dans les prairies couvertes de jeunes herbes.

Ces mesures permirent de diminuer les pertes, mais il ne fut pas possible de supprimer l'évolution des symptômes décrits. Toutefois, l'application massive de la solution cupro-arsénicale parvenait à maintenir assez péniblement le bétail jusqu'en saison sèche, époque où son état général s'améliorait considérablement. Comme l'action antiparasitaire du médicament se révélaif fort douteuse, on pouvait penser que son efficacité était due à une autre action que l'action parasificide. L'apport de cuivre ou d'impuretés compensait-il une carence minérale ? C'est pour

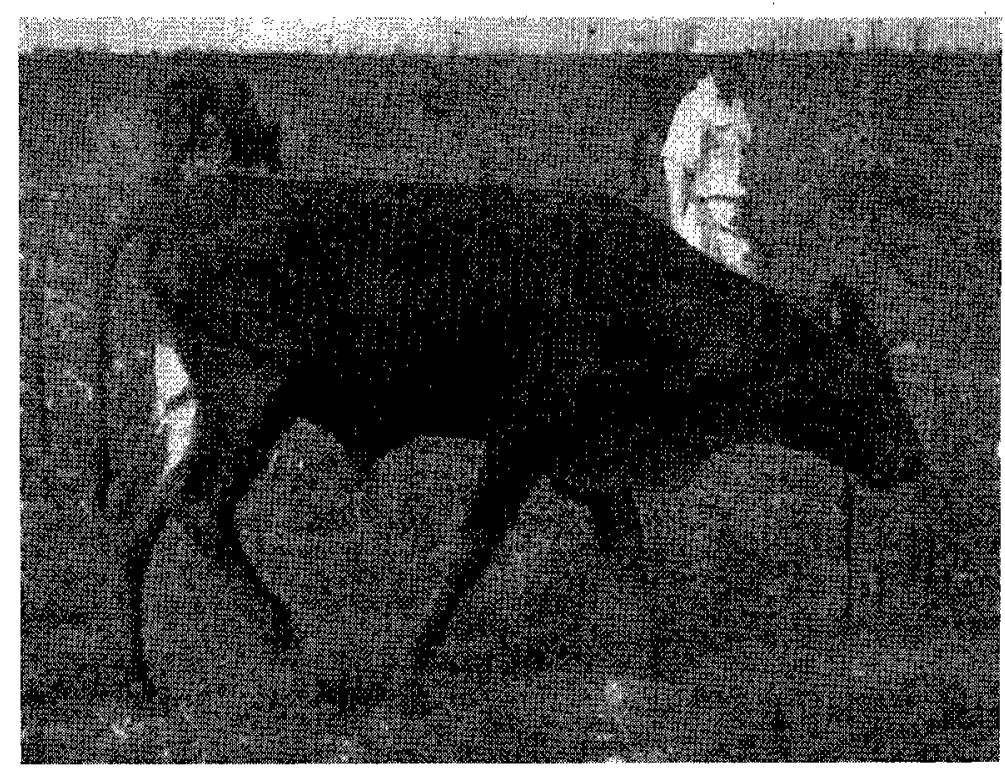

Photo 3. - Bouvillon d'un an et demi montrant un stade plus avancé de la maladie. 
élucider cette hypothèse que nous avons entrepris notre expérimentation.

\section{EXPÉRIMENTATION}

1. Analyse du fourrage, au point de vue des oligo-éléments.

a) provenant de pâturages suspects.

b) provenant de pâturages normaux.

2. Etude comparative sur 4 lots de bouvillons

Chaque lot fut composé de 30 animaux en bonne santé, avec

10 bêtes de 1 an $1 / 2$.

10 bêtes de 2 ans $1 / 2$.

10 bêtes de 3 ans $1 / 2$. 56.

Durée de l'expérience : du 25-9-55 au 22-5-

Pesée des animaux une fois par mois, à jeun.

Les bêtes furent marquées au fer rouge et ont pâturé sur les mêmes prairies réputées douteuses pour les lots 1-2 et 3 ; le lot 4 a pâturé sur des prairies réputées normales.

Lot no 1.

Le groupe témoin a reçu une fois par semaine la même quantité de $5 \mathrm{~kg}$ de sel ordinaire, pour les 30 animaux, que les lots 2,3 et 4 , mais sans aucun supplément. Le sel fut distribué dans de longs bacs en bois, le soir, dans le kraal.

Lot $n^{\circ} 2$.

On ajoute $8 \mathrm{~g}$ du mélange d'oligo-minéraux par $100 \mathrm{~kg}$ de bétail sur pied et par semaine.

Composition par $\mathrm{kg}$ du mélange d'oligo-minéraux :

$\mathrm{Mn}: 48 \mathrm{~g}, \mathrm{Fe}: 46 \mathrm{~g}, \mathrm{Mg}: 8 \mathrm{~g}, \mathrm{Cu}: 1.4 \mathrm{~g}$ $\mathrm{I}: 3 \mathrm{~g}$, Co: $0.36 \mathrm{~g}$. Zn: $0.15 \mathrm{~g}$.

La quantité de cobalt distribuée fut donc:

$0.41 \mathrm{mg}$ par jour par $100 \mathrm{~kg}$ de bétail sur pied.

Lot $n^{\circ} 3$.

On ajoute du sulfate de cobalt à raison de :

$0,6 \mathrm{mg}$ de cobalt par jour par $100 \mathrm{~kg}$ de bétail sur pied.

Lot no 4.

Groupe témoin, soumis aux mêmes conditions que le lot $n^{0} 1$, mais pâturant sur des prairies réputées normales à sol riche en limonite.
Durée de l'expérience: De septembre 1955 à mai 1956 inclus.

\section{RÉSULTATS}

\section{Fourrage :}

(Les analyses ont été faites sous la direction du Professeur L. HENNAUX, à l'Institut Agronomique de l'Etat à Gembloux).

Analyses 1953/1954, moyenne de 4 échantillons (en $\mathrm{mg}$ par $\mathrm{kg}$ de matière sèche).

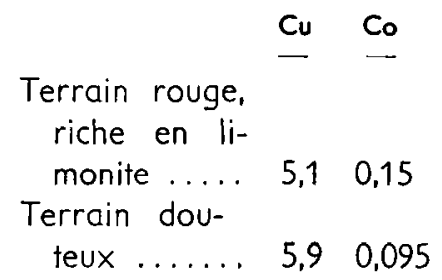

Analyses 1955/1956, moyenne de 13 échantillons :

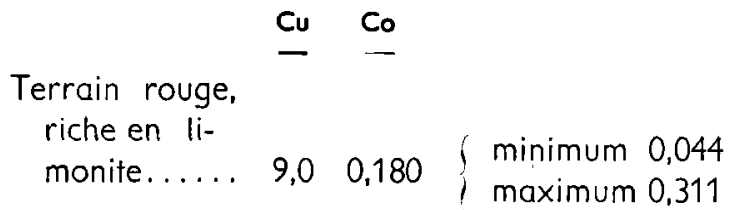

$\begin{gathered}\text { Terrain dou- } \\ \text { teux } \ldots \ldots\end{gathered} \quad 9,4 \quad 0,142 \quad \begin{aligned} & \text { minimum } 0,063 \\ & \text { maximum } 0,210\end{aligned}$

HENNAUX (6) a trouvé, sur 16 échantillons provenant de différents endroits du Katanga, une moyenne de 21,5 pour le $\mathrm{Cu}$ et de 0,8 pour le Co en $\mathrm{mg}$ par $\mathrm{kg}$ de matière sèche.

Les valeurs trouvées pour les autres éléments analysés ne sont pas rapportées; elles peuvent être considérées comme normales pour le P-Ca$\mathrm{Mg}-\mathrm{K}-\mathrm{Na}-\mathrm{Fe}$ et $\mathrm{Mn}$.

\section{Interprétation :}

Les taux du Cu peuvent être considérés comme bas, mais encore dans les limites normales.

Les taux de Co sont limites et légèrement inférieurs dans les herbages provenant de prairies considérées comme dangereuses, par rapport à celles considérées comme saines. Toutefois les taux trouvés ne permettent pas de les classer avec certitude parmi ceux qui provoquent des symptômes de carence, si l'on se refère aux chiffres cités par HASLER et ZUBER (4). 

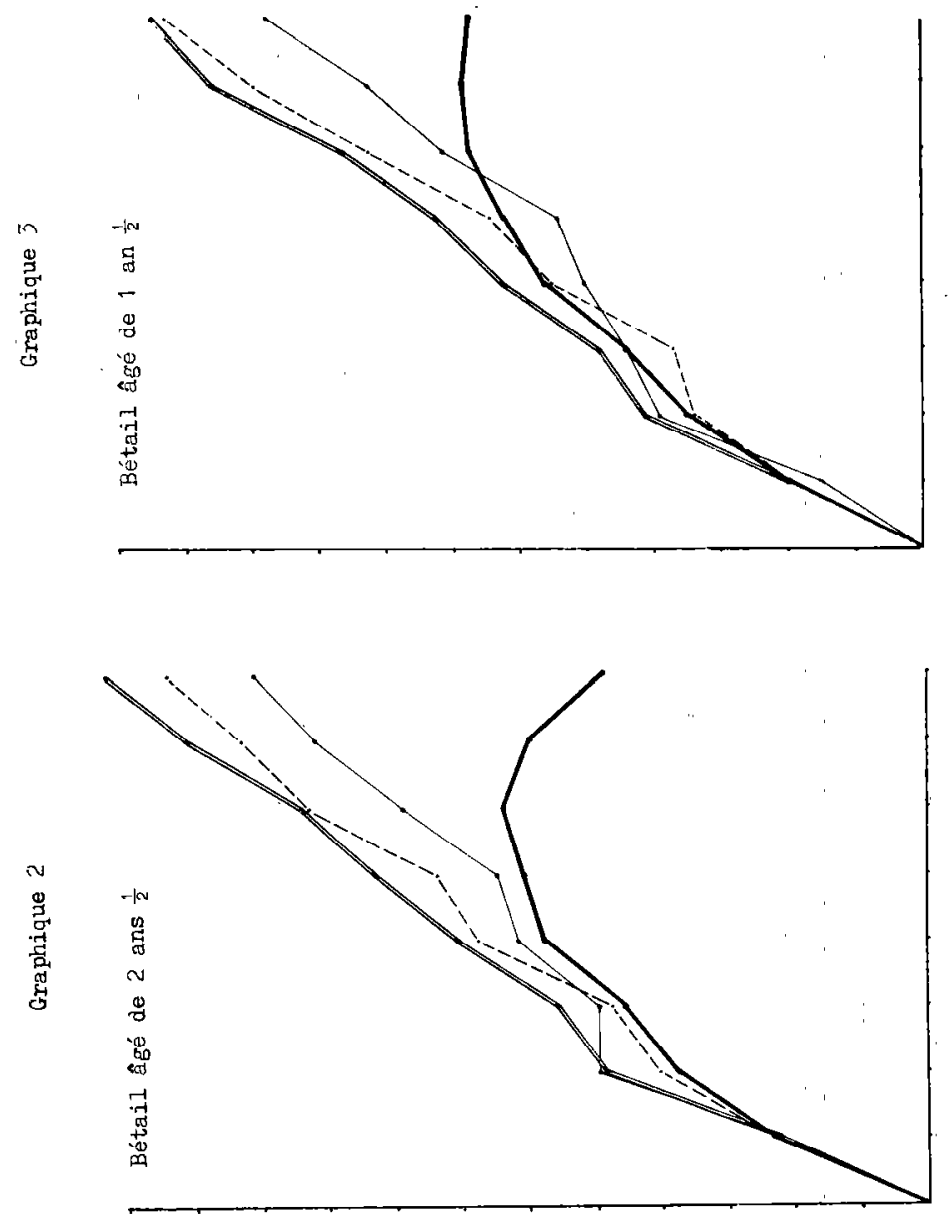

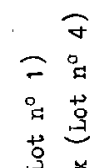

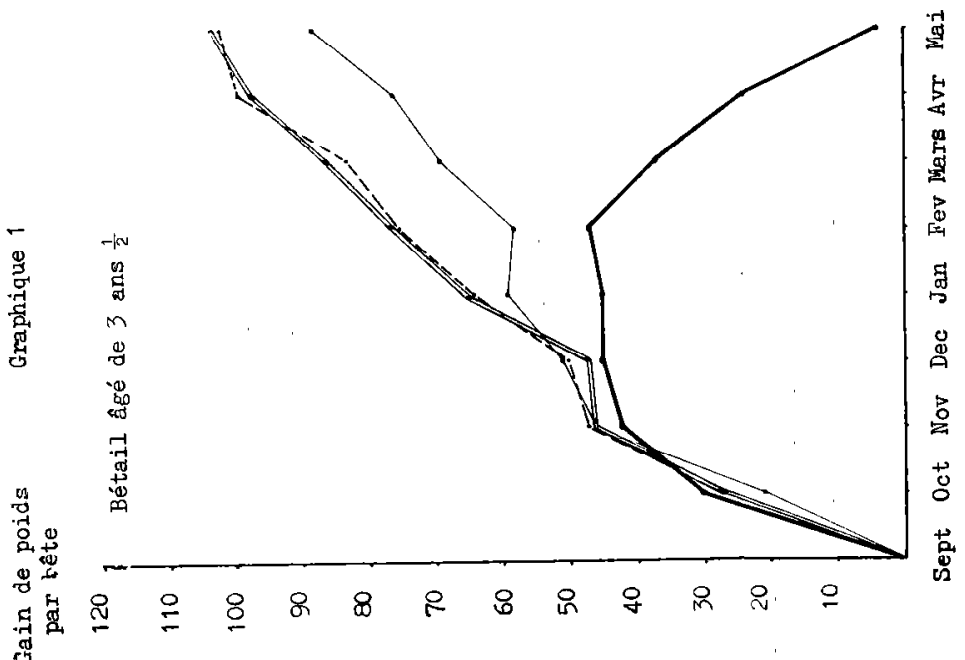

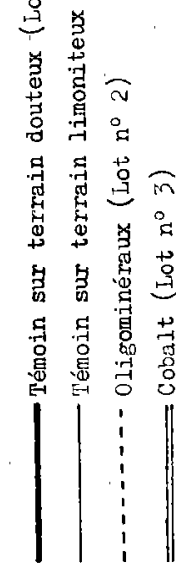

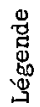




\section{Animaux :}

Les résultats des pesages sont consignés dans le tableau 1 ci-après :

Les graphiques 1,2 et 3 expriment les différences de poids en fonction du temps (du mois de septembre 1955 au mois de mai 1956), en tenant compte de l'âge de chaque lot d'animaux.

\section{Interprétation :}

L'adjonction d'oligo-éléments et surtout de sel cobaltisé a une action indiscutablement mise en évidence. Toutefois quelques remarques s'imposent :

1. Les courbes des différents groupes d'âge du lot témoin ne reflètent pas l'état général réel du bétail. Les animaux de 1 an 1/2 ont terminé la saison des pluies en très mauvais état ; 3 bêtes sont mortes ou ont dû être enlevées du troupeau et traitées. La courbe relativement favorable de ce jeune bétail, par rapport aux courbes des animaux plus âgés du même lot, s'explique par une augmentation de poids due à la croissance du squelette des jeunes bêtes.

$\mathrm{Ce}$ facteur de croissance intervient moins dans le groupe d'âge de 2 ans $1 / 2$ et presque plus dans celui de 3 ans 1/2. La courbe du groupe de bétail le plus âgé est donc la plus fidèle pour exprimer l'évolution de l'état général.

2. Tous les animaux du lot no 1 ont été atteints, durant la deuxième moitié de la saison des pluies, des symptômes décrits au paragraphe II. Les animaux des lots 2, 3 et 4 sont restés en excellent état.

3. Les courbes des animaux recevant les oligo-éléments ef le sel cobaltisé expriment la même évolution favorable. On peut conclure que le cobalt seul est capable de supprimer la maladie et que les autres oligo-éléments ne sont pas nécessaires à une telle évolution.

4. La courbe du lot no4 (pâtures saines) exprime une évolution favorable parallèle, mais plus lente que celle des lots 2 et 3 . On peut supposer que la teneur en cobalt des herbages est irrégulière ou inférieure à la teneur optimum, mais une affirmation dans ce sens devrait être confirmée par une autre série d'essais.

\section{CONCLUSIONS}

1. Le sel cobaltisé, mélangé au sel ordinaire, a protégé les animaux contre les troubles décrits.
La symptomatologie observée peut donc être considérée comme une carence en cobalt. 0,095 d̀ $0,142 \mathrm{mg}$ de Co par $\mathrm{kg}$ de matière sèche des herbages (moyennes des pâturages suspects) n'ont pas protégé les bovidés des troubles de carence, dans les conditions locales de l'expérimentation.

2. L'adjonction de $0.41 \mathrm{mg}$ de cobalt, par $100 \mathrm{~kg}$ de bétail sur pied, et par jour, représente une dose efficace pour protéger le bétail contre les troubles de carence.

L'administration hebdomadaire s'est avérée suffisante.

3. L'expérience décrite n'explique pas, mais constate que les troubles de carence sont liés à la saison des pluies.

MARSTON (8) (d'après GEE) signale que la teneur en cobalt des herbages varie suivant les saisons et les années. Nous pouvoris penser que cette explication s'accorde avec nos observations.

En outre, en saison sèche, le bétail est obligé de pâturer sur de très grandes étendues ( $4-5$ hectares par tête contre 1-2 hectares en saison de pluie). Les animaux sont obligés de suivre des fonds herbeux qui restent plus humides. On peut supposer que ces pâtures, plus acides et où le terrain est plus alluvionnaire, contiennent davantage de cobalt et permettent ainsi aux animuux de se maintenir en meilleur état.

\section{Applications pratiques.}

L'administration supplémentaire de cobalt a été appliquée dès lors à l'ensemble du bétail, sans tenir compte du terrain des pâturages.

Les résultats de cette adjonction furent surprenants :

1. Les pâturages carencés, qui avaient dû être abandonnés temporairement en saison de pluies, purent de nouveau être occupés en 'permanence.

2. Le nombre de têtes par unité de superficie a pu être augmenté et le poids moyen de boucherie s'est sensiblement amélioré.

3. La rotation des pâturages, visant à ne donner au bétail que de jeunes pousses à brouter. a automatiquement subi certaines modifications. Les animaux cobaltisés réussirent à les utiliser mieux en broutant également les herbes plus vieilles.

Cette aptitude peut s'expliquer par une meilleure digestion d'une nourriture relativement plus riche en cellulose, digestion rendue possible 
TABLEAU NO 1

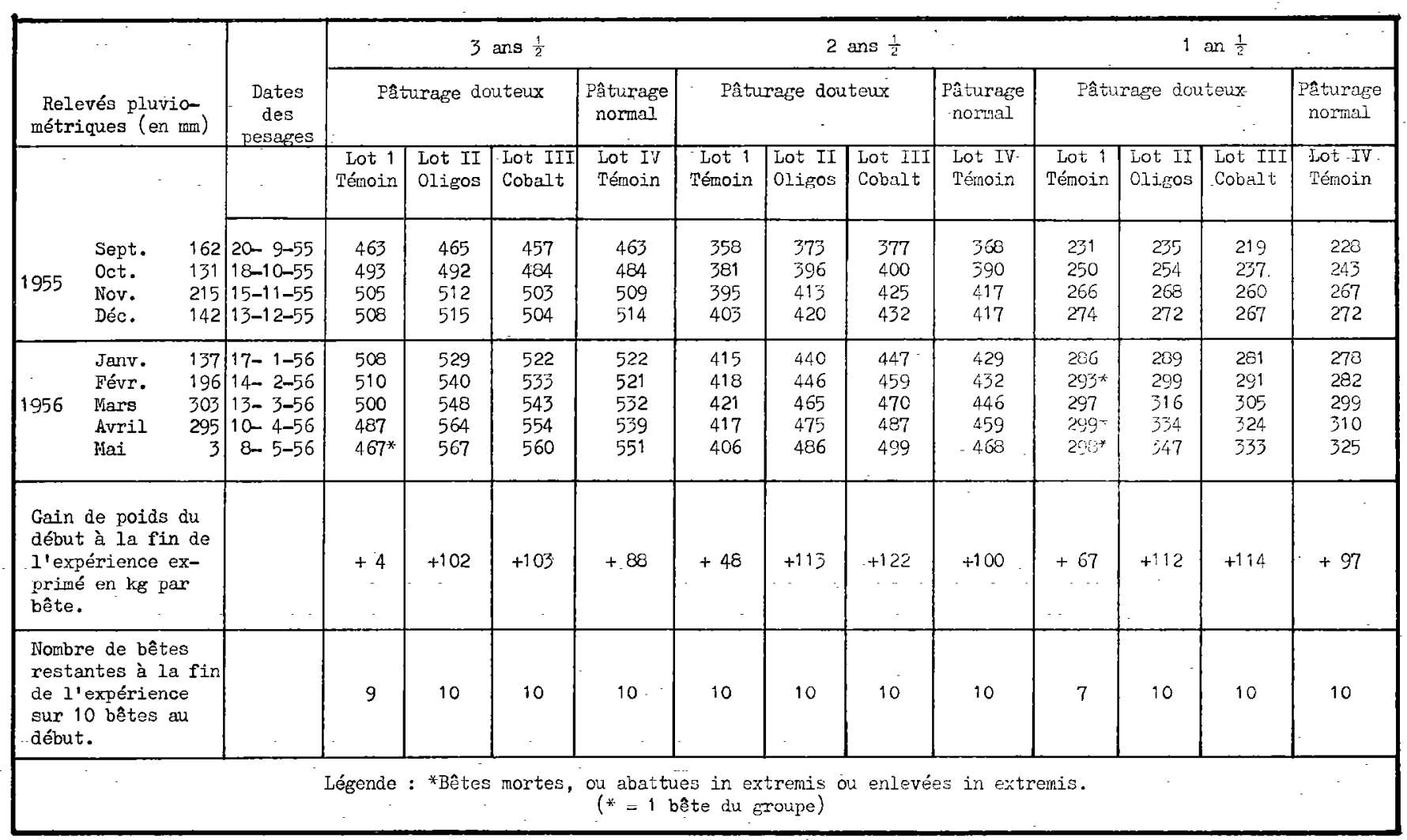


parce que la flore intestinale devient vraisemblablement plus variée et plus complète sous l'influence de l'apport supplémentaire de cobalt.

Jétiens à remercier particulièrement :

le Docteur E. LUTZ de m'avoir procuré la possibilité d'effectuer les expériences décrites, le Professeur L. HENNAUX, quii a effectué les analyses d'herbes à l'institut Agronomique de l'Ełat à Gembloux,

Monsieur C. HENROTIN qui a collaboré efficacement à l'exécution de notre expérimentation,

le Docteur C. LAMBERT qui a fait la supervision du texte, ainsi que les nombreux collaborateurs européens et africains qui m'ont aidé dans ce travail.

\section{RÉSUMÉ}

Nos recherches ont démontré que la déficience en cobalt dans l'herbe de certains pâfurages du Katanga se manifeste sur les bovidés par les symptômes principaux suivants :

Perte d'appétit - pica - chute pondérale - anémie - diarrhée.

Des additions de cobalt de l'ordre de $0,41 \mathrm{mg}$ par jour et par $100 \mathrm{~kg}$ de poids vif ont été suffisantes pour supprimer chez les bovins l'apparition et l'évolution des symptômes de carence. L'administration de cobalt, une fois par semaine, mélangé au sel ordinaire, fut pleinement satisfaisant. Le cobalt favorise l'existence et l'action de la flore physiologique du rumen. Cette dernière rend possible la digestion d'une nourriture riche en cellulose.

La conclusion pratique de notre travail se résume à la mise en évídence de l'importance d'un apport supplémentaire de cobalt afin d'obtenir une meilleure exploitation des pâturages carencés. II permet de ce fait d'augmenter le nombre de bêtes par unité de superficie et le poids du bétail de boucherie.

\section{SUMMARY}

\section{Cobalt deficiency in Cattle Ranching in Katanga}

Investigations have shown that the cobalt deficiency in the grasses of some parts of Katanga result in the following symptoms in cattle: anorexia, pica, loss of weight, anaemia, and diarrhoea.

A daily supplement of $0.20 \mathrm{mg}$. of cobalt for each eut of live-weight is adequate to prevent the appearance and development of the deficiency syndrome. Weekly administration of cobalt combined with common salt proved absolutely adequate. Cobalt encourages the presence and activity of the rumen flora which favours the digestion of cellulose.

The author concludes that cobalt additive is important to correct this pasture deficiency. By its use the carrying capacity of the land would be increased as also the individual carcase weights.

\section{RESUMEN}

\section{Carencia de cobalto en una explotacion de bavidos en Katanga}

Nosotros hemos encontrado que la deficiencia en cobalto de la hierba de ciertos pastizales de Katanga, se manifiesta en los bovidos por los principales sintomas siguientes : perdida de apetito. pica, perdida de peso, anemia, y diarrea.

La administracion de cobalto, a la dosis de $0,41 \mathrm{mg}$ por $100 \mathrm{~kg}$ de peso vivo, diarios, ha sido suficiente para evitar en los bovidos la aparicion y evolucion de los sintomas de carencia. La adiccion de cobalto una vez por semana, mezclada a la sal ordinaria, fue plenamente satisfactoria. El cabalto fovorece la existencia y la accion de la flora fisiologica del rumen y esta ultima hace posible la buena digestion de una alimentacion rica en celulosa. 


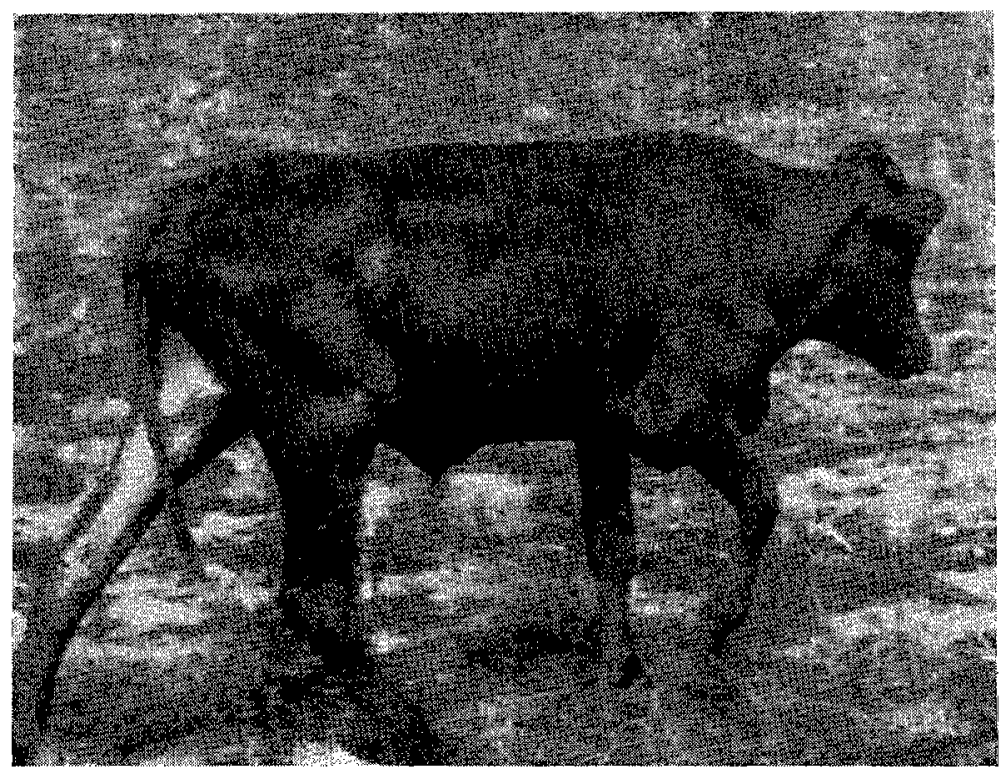

Pholo 4. - Le même animal malade...

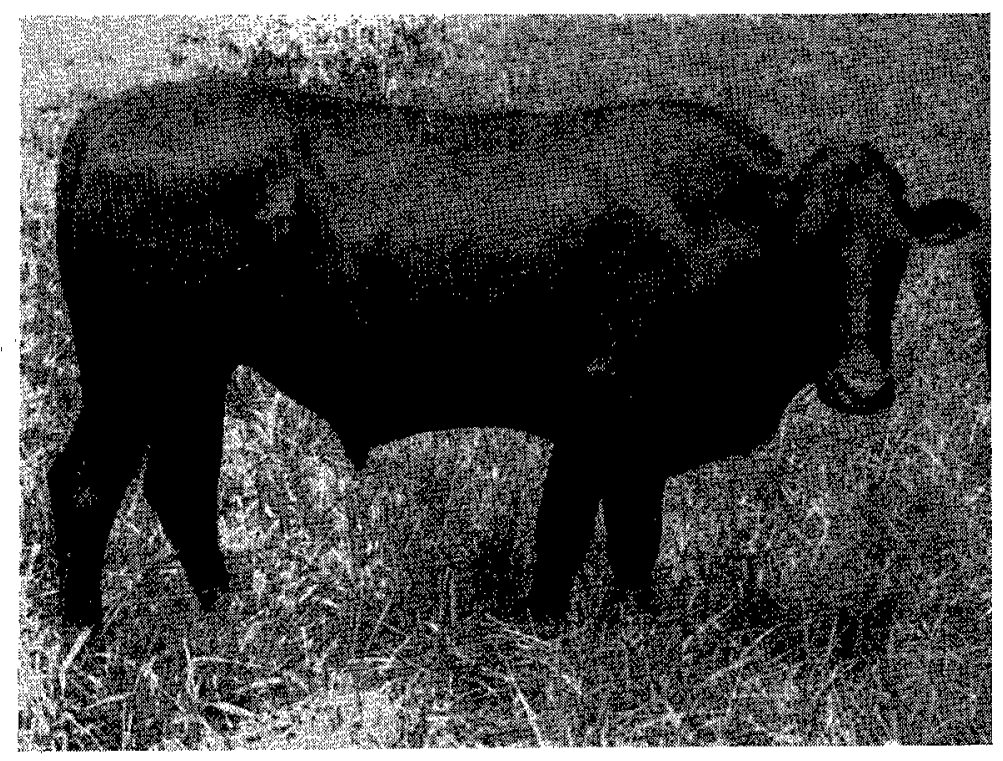

Photo $5 .-\ldots$ et rétabli après traitement au cobalt. 
Nuestras conclusiones practicas se reducen a la impotancia que tiena un aporte suplementario de cobalto a fin de obtener mejores resultados en el aprovechamiento de pastizales con carencia. Pues ello permite aumentar el numero de animales por unidad de superficie $y$ el peso del ganado para carne.

\section{ZUSAMMENFASSUNG}

Unsere Untersuchungen haben gezeigt, dass der Mangel an Kobalt im Gras von gewissen, hier in Frage stehendenWeiden des Katanga, sich beim Rind in folgenden Hauptsymptomen âussert:

Appetitverlust-Pica-Gewichtsverlust-Anaemie-Durchfall.

Die zusätzliche Gabe von $0,41 \mathrm{mg}$ Kobalt pro Tag für $100 \mathrm{~kg}$ Lebendgewicht haben beim Rind genügt, um das Auftreten und die Entwicklung von Mange lerscheinungen zu unterbinden. Die Verabreichung kann einmal wốchentlich, vermischt mit gewöhlichem Salz, erfolgen.

Das Kobalt begünstigt die Lebensmoglichkeiten der Pansenflora, welche ihrerseits die Verdauung von zellulosereicher Nahrung im Rumen ermóglicht.

Die praktische Schlussfolgerung unserer Arbeit besteht im Hinweis auf die Bedeutung der zusâtzlichen Verabreichung von Kobalt zur besseren Ausnützung von zweifelhaften Weidegebieten. Sie führt zur Hebung der Tierzahl pro Oberflächeneinheit und zur Verbesserung des Durchschnittsgewichtes der Schlachttiere.

\section{BIBLIOGRAPHIE}

1. BENDIXEN (H.C.). - Kobaltmangelkrankheit der Wiederkäuer-Erfahrungen in Dänemark. Dtsch. Tierärztl. Wch. 1961, 8.

2. BEESON (K. C.). - U. S. Dept. Agric. Inf. Bull. 1950, 7, cité par Gee. (R. W).

3. GEE (R. W.). - Cobalt and ruminant nutrition. Publication of Nicholas Proprietary Ltd., oct. 1958, Melbourne, Australia.

4. HASLER (A.), ZUBER (R.). - Beitrag zur Kenninis des Kobaligehaltes im schweizerischen Wiesenfutter. Schweiz. Landwirtschaft. Mh., 1955, 33 (5).

5. HENNAUX (L.). - L'Alimentation minérale du bétail au Congo belge. Académie royale des sciences coloniales, 1956, 3. (48) (série technique).

6. HENNAUX (L.). - Communication personnelle.

7. LUTZ (E). - Contribution à l'étude des maladies parasifaires internes des bovidés. Rev. Elev. Méd. Vét. Pays trop. 1948, 3, 165-74.

8. MARSTON (H. R.). - Brit. Commonwealth Scientific Official Cont. (Australia), 1949, London. H. M. S. 0. 1957 (cité par Gee R.W.).

9. SCHARRER (K.). - Biochemie der Spurenelemente. Parey, Berlin/Hamburg. 1955. 